\title{
Encapsulate this: the do's and don'ts
}

\section{To the Editor:}

In the January News Feature "Encapsulate this," Elie Dolgin summarizes recent efforts by a team of bioengineers at the Massachusetts Institute of Technology (MIT) to use a new high-throughput screening approach to identify a biomaterial for islet encapsulation that can avoid the host's immune response $\mathrm{e}^{1}$. The article is important, and some aspects it highlights, such as human trials, are timely. However, we would argue that it focuses mostly on obstacles that the field solved some time ago. It introduces new solutions to old problems that are no more beneficial than already existing approaches, such as purification of alginates. If used adequately, alginates do not provoke fibrotic tissue responses for the life span of experimental animals ${ }^{2}$, and, in humans, their safety and biocompatibility have been shown to last for very long periods of time ${ }^{3,4}$. Furthermore, some of the advances reported in the article, such as the identification of the first new alginate that escapes immune attack, and the effects of islet size and loads, have been previously identified and discussed by other scientists working in the field 5 .

The article states that all attempts at islet encapsulation in the absence of immunosuppression have failed. This is an overstatement that is simply not true. Many groups have been successful in transplanting islets in the absence of immunosuppression in experimental animals, including large mammals and humans ${ }^{3,6}$. These studies were done with immunoprotective capsule types such as alginate-poly-L-lysine capsules and alginate-poly-L-ornithine capsules. We feel this lack of immunoprotection should have been mentioned to avoid false hope for patients who are often desperately seeking better alternatives for insulin therapy.

To suggest that no systematic approaches have been applied and that researchers are simply randomly trying different things ignores all of the breakthroughs of the past decade by many scientists in the field. Alginate biocompatibility is a widely discussed topic in the literature. In the past decade, there are at least 20 comprehensive articles describing how alginates can be optimized, purified and tailored to enhance their beneficial properties and potential as key biomaterials for fabricating three-dimensional alginate capsules. However, in cell microencapsulation, the concept of alginate biocompatibility cannot be limited to the alginate and certainly not by the approaches described. Other key parameters include the type of immunoprotective coating, the type of crosslinking ions, the type of the enclosed cell, the geometry, smoothness and charge of the surface of the capsules, the microencapsulation technology and the diameter of the final capsules. Dolgin's article considers only the starting material, with a brief note that capsule size can matter.

In conclusion, we feel that the News Feature gives an incomplete overview and understates the current status of the field. The encapsulation field has advanced considerably over the past 20 years to the point that it represents a viable therapeutic option worthy of clinical evaluation. The current true challenges, such as finding better transplantation sites, improving nutrition of the encapsulated tissue, avoiding lab-to-lab variations and controlling crucial surface parameters, are ignored. We would also like to point out that guidelines to address key capsule properties that are missing from the News Feature have been published by a European consortium ${ }^{7}$.

\section{COMPETING FINANCIAL INTERESTS}

The authors declare competing financial interests: details are available in the online version of the paper (doi:10.1038/nm.3486).

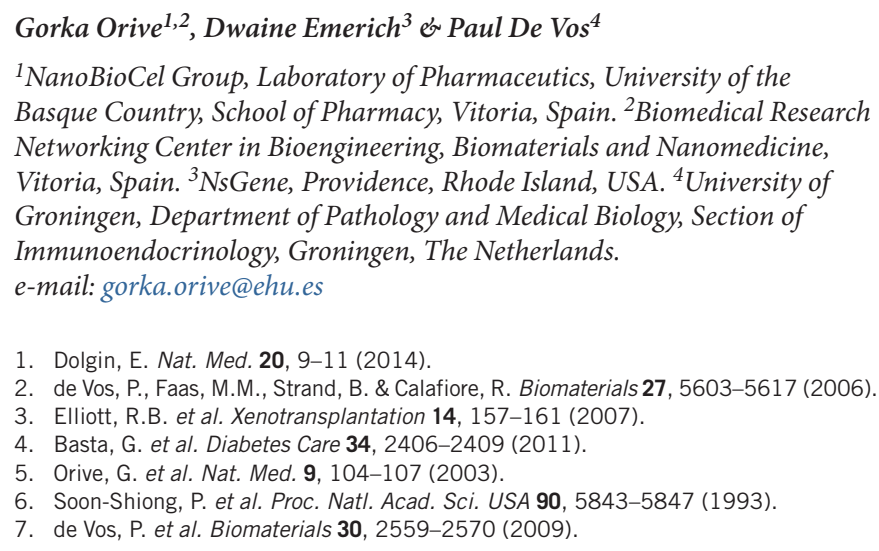

\section{Nature Medicine replies:}

We thank Orive and his colleagues for their thoughful argument that existing materials for islet encapsulation are suitable for therapeutic applications ${ }^{1}$. However, this does not seem to be the opinion of most scientists working in the field of islet encapsulation, as evidenced by the comments and opinions outlined in the News Feature ${ }^{2}$. One notable proponent of the need for improved biomaterial barriers is the JDRF, which handed out one of the largest academic grants ever in this discipline specifically for the pursuit of a new alginate-based material. As discussed in the News Feature, this JDRF-funded project is beginning to bear fruit, with preliminary results showing that the new alginate derivate is superior to previously tested materials. Given the dearth of promising data on the application of islet encapsulation in humans or other primates, Nature Medicine maintains that this is a worthy and exciting area of research to highlight in our News pages.

1. Orive, G., Emerich, D. \& De Vos, P. Nat. Med. 20, 233 (2014).

2. Dolgin, E. Nat. Med. 20, 9-11 (2014). 